\title{
Enfermeras anestesistas años 1970 a 1980, hospitales terciarios: rol del profesional
}

DOI: $10.46981 / \mathrm{sfjhv2n2-001}$

Received in: January 1st, 2020

Accepted in: March 30th, 2020

\section{Verónica San Martin Osses}

Enfermera, Magister en Enfermería Mención Gestión del Cuidado, Docente Escuela de Enfermería, Universidad Mayor sede Temuco. Temuco. Chile.

E-mail: veronicasanm@gmail.com

\section{Dra. Edith Rivas Riveros}

Enfermera, Doctora en Enfermería, Docente Dpto. de Enfermería, Universidad de La Frontera. Temuco. Chile.

E-mail: edith.rivas@ufrontera.cl

\section{RESUMEN}

Introducción: el rol profesional desempeñado por las enfermeras anestesistas en los hospitales terciarios de Chile, no ha sido reconocido ni documentado, debido a esto se realiza la presente investigación histórica relacionada con el tema. Objetivo: develar el rol de las enfermeras en el área de anestesia, en los años 1970 a 1980, en un hospital del sur de Chile. Método: estudio histórico, a través de historia oral, que incorpora en la recolección de información, fotografía de época y entrevista no estructurada. Análisis de contenido e iconográfico. Cumple criterios éticos según Exequiel Emmanuel (1) y consentimiento informado. Resultados: debido a la falencia importante de médicos anestesiólogos, fue necesaria la preparación de enfermeras en anestesia. Quienes, por su preparación y dedicación, fueron un pilar fundamental en el desarrollo del área quirúrgica por años en hospitales de menor complejidad.

Discusión: se destaca la importancia que tuvieron a nivel de salud nacional, en el transcurso de la historia, las enfermeras anestesistas, demostrando entrega y compromiso en su labor, desarrollando una función médica con alto grado de profesionalismo, a pesar de haber sido subvaloradas y poco reconocidas en el desarrollo de la cirugía en Chile.

Conclusión: el trabajo de las enfermeras anestesistas permitió la resolución quirúrgica de numerosas intervenciones en hospitales terciarios, siendo un aporte importante a la salud, hecho histórico, poco reconocido y valorado.

Palabras clave: Enfermería, Historia de la enfermería en Chile o Enfermera anestesista. DeCS.

\section{INTRODUCCIÓN}

La formación universitaria de médicos anestesistas en Chile comienza en el año 1963 en la Universidad de Concepción. A partir de 1965, se incorpora esta especialidad en otras universidades del país. La anestesiología se define como una especialidad del área médica, dedicada al confort y cuidados de los pacientes durante la intervención quirúrgica y otros procesos que puedan resultar molestos o dolorosos; su rol principal es preocuparse de mantener la hemodinamia del paciente en rangos normales, profundidad anestésica, adecuado alivio del dolor y bienestar general de éste. (2) En lo que respecta a la 
dotación de médicos anestesistas en el país, recién a fines de 1980 se logra la formación de un número considerable de estos especialistas los cuales fueron distribuidos en distintos hospitales de Chile. (3)

Es por esta falencia de anestesiólogos que se preparan enfermeras en anestesia, las cuales eran capacitadas en el Hospital del Salvador, en Santiago de Chile (hospital de primer nivel), provenientes en su mayoría de hospitales regionales. Es así como, en el hospital de estudio, fueron capacitadas cuatro enfermeras que trabajaban en el servicio de pabellón, quienes asumieron el rol de enfermeras anestesistas.

La investigación se realiza con la intención de develar el rol profesional de enfermeras anestesistas, que trabajaron en el período antes mencionado, al ejercer una función definida en la literatura como propia de los médicos.

El desarrollo de la investigación se realiza a través de cuatro entrevistas a una enfermera, que trabajó en el período de estudio, junto a la revisión de documentos de época facilitados por una segunda enfermera de la época.

\section{OBJETIVO GENERAL}

Develar el rol profesional de enfermeras en el área de anestesia, en el servicio de pabellón central de un hospital de nivel terciario al sur de Chile, en el periodo de 1970 a 1980.

\subsection{OBJETIVOS ESPECÍFICOS:}

- Identificar el rol de enfermería como enfermera anestesista en el servicio de pabellón de un hospital de nivel terciario, durante el período en estudio.

- Revisar importancia de enfermería en la administración de anestesia en el servicio de pabellón de un hospital de nivel terciario, durante el período en estudio.

- Describir el respaldo legal de enfermeras que administraron anestesia en el servicio de pabellón de un hospital de nivel terciario, durante el período en estudio.

\section{MATERIAL Y METODO}

Se utilizó el método analítico-sintético, analizando los sucesos ocurridos en el período entre 1970 a 1980 en Chile, en un hospital de nivel terciario, a nivel de enfermería, con una posterior síntesis de los hechos históricos. (4)

Por otra parte, en lo que respecta a la historia de la anestesia en enfermería, en la segunda mitad del siglo XIX, las enfermeras asumen este rol debido a la escasez de médicos anestesistas, (5) manteniendo su formación de base que era el cuidado integral del individuo, familia y comunidad en todas las etapas del ciclo vital. (6) 
Ya en la segunda guerra mundial se consolida su rol en anestesia, incorporándolas a la estructura militar de los EEUU, declarándolo como especialidad en el campo clínico, posteriormente, en el año 1952 se formaliza a través de un proceso de certificación. (7) En el caso de Europa, después de la segunda guerra mundial, se recurrió a enfermeras anestesistas que trabajaban bajo la supervisión de un anestesiólogo y en países como Francia y Holanda se imparte un posgrado en ésta área. (8)

Por otro lado, en relación a la recolección de datos, se realizó a través de entrevista no estructurada, entendiéndose que es aquella que se basa en una guía general del contenido, en donde el entrevistador tiene la flexibilidad para manejarla. (9) Contactando y entrevistando, de esta forma, a una de las enfermeras involucradas en la investigación, utilizando como apoyo una grabadora y libreta de anotaciones. Una vez recolectada la información se realiza transcripción y análisis lineal. Además, se complementa con revisión bibliográfica y material fotográfico.

Para la obtención de información se aplica un consentimiento informado a profesional entrevistada.

En lo que respecta al sujeto de estudio, es una enfermera egresada de la Universidad de la Frontera de Chile, quién desde el año 1970 se desempeñó profesionalmente en el servicio de pabellón en el hospital de estudio, realizando el rol de enfermera anestesista hasta 1980.

\section{RESULTADOS}

Se muestra un cuadro de resultados, con las siguientes categorías: formación de enfermeras en anestesia y rol de enfermeras anestesistas.

Cuadro 1.

\begin{tabular}{|l|l|}
\hline Categorías & Subcategorías \\
\hline Formación de enfermeras en anestesia & $\bullet \quad$ Déficit de médicos anestesistas en el país \\
& $\bullet \quad$ Formación impuesta por la institución \\
\hline Rol de enfermeras anestesistas & $\bullet \quad$ Rol asistencial \\
& $\bullet \quad$ Trabajo en equipo \\
& $\bullet \quad$ Compromiso profesional \\
& $\bullet \quad$ Manejo de pacientes de alta complejidad \\
\hline
\end{tabular}


La primera categoría, en sus subcategorías evidencia que la capacitación surge por un déficit de médicos anestesistas en el país y la región, debido a lo cual, se decide como política de salud, capacitar a enfermeras y matronas en el área de anestesia. "partió por la necesidad de incluir enfermeras en anestesia. Para los técnicos era demasiada responsabilidad su administración. Además, había solo dos médicos anestesiólogos los cuales estaban contratados por muy pocas horas, así que la mayor parte del tiempo el servicio funcionaba con enfermeras y técnicos." (entrevista 1)

También surgen otras subcategorías como, formación impuesta por la institución realizándose en un hospital de primer nivel, "entonces, nos dijeron que teníamos que ir, yo llevaba poco tiempo trabajando. No nos preguntaron, fuimos cuatro enfermeras a Santiago, al hospital del Salvador, donde nos enseñó el anestesista jefe, nos hacían clase en una sala del hospital y luego practicábamos en pabellón, donde nos enseñaron todas las técnicas de anestesia”. "Estuvimos alrededor de 10 meses allá capacitándonos". "Cuando llevábamos algunos años trabajando, formamos cuatro enfermeras más, más o menos en el año 1976, de esta forma todos los pabellones podían funcionar". "Creo que a nivel nacional, fue una necesidad formar enfermeras en anestesia, ya que los médicos en general eran muy pocos". (entrevistas 1,2 y 3 )

En el caso de la segunda categoría, se evidencian subcategorías como que el rol asistencial, existencia de trabajo en equipo, alto compromiso con el paciente y la institución, y manejo de pacientes de alta complejidad; refiriendo la entrevistada: "recibiamos el paciente, se revisaba la ficha, haciendo la anamnesis, y luego se ingresaba a pabellón. No existía tanta tecnología como ahora, manualmente tomábamos los signos vitales, el control del pulso lo hacíamos durante toda la cirugía, desarrollando mucho la observación". "Todo se hacía manual, incluso ventilar al paciente, por ejemplo la máquina de anestesia tenía la bolsa para ambusear así que la mano era el ventilador". "Trabajábamos solas, sin un médico supervisándonos, la cirugías no tan complejas nos ayudaban las técnicos que se habían preparado en procedimientos básicos de anestesia, pero los enfermos graves solo nosotras nos hacíamos cargo." "Yo jamás tuve un problema o complicación con un paciente, pero si ocurría una emergencia como un parao cardio respiratorio, el médico cirujano se hacía responsable”. (entrevista $1,3$ y 4$)$

Así pues, este grupo de enfermeras en conjunto con los dos médicos anestesiólogos, y ocasionalmente con el apoyo de auxiliares de enfermería, trabajaron en equipo por alrededor de 10 años, formaron cuatro enfermeras más para resolver la necesidad quirúrgica de la época. Ya en 1980, se incorporaron dos médicos anestesiólogos, tomando las cirugías de mayor complejidad, y en forma gradual desplazando el trabajo de enfermería. 
Alrededor del 1990, se incorporaron cuatro anestesiólogos más, desplazando definitivamente el trabajo de enfermería. A partir de ésta fecha fueron ellos quienes tomaron en su totalidad la anestesia. Las enfermeras fueron reasignadas tanto en pabellón como dentro de la institución.

\section{ANÁLISIS ICONOGRÁFICO}

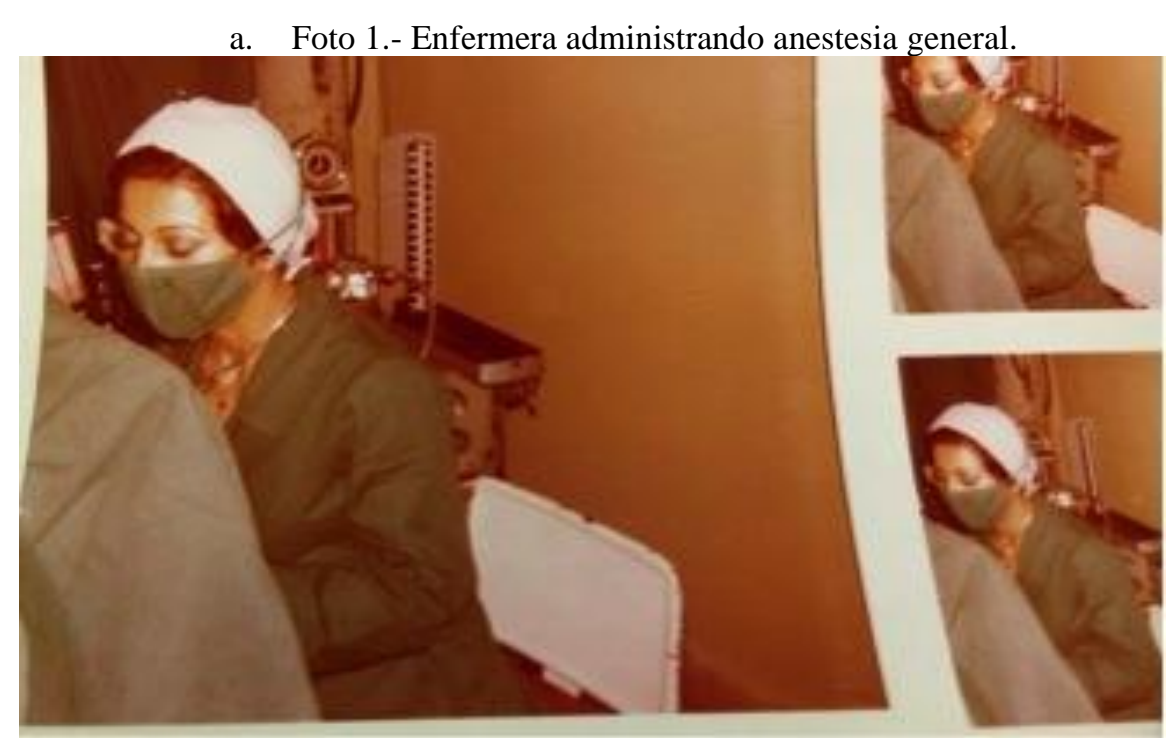

\subsection{PROCEDIMIENTO ESQUEMÁTICO:}

- Autor: enfermera de la época.

- Lugar y fecha de realización: hospital de estudio, sala quirúrgica, año 1976.

- Tipo de imagen: fotografía

- Contexto histórico: fotografía tomada en el año 1976, cuando la enfermera desempeñaba su rol de anestesista, en un hospital de nivel terciario, en Chile.

- Descripción de elementos: fotografía a color, se aprecia a la enfermera de la época administrando anestesia general a paciente en medio de una intervención quirúrgica, se distingue uniforme de color verde, y gorro de género de color blanco, ropa quirúrgica igual que el uniforme. Además se aprecia una silla metálica para realizar el procedimiento. Al fondo de la fotografía se puede visualizar un esfigmomanómetro de mercurio, cuyo funcionamiento era manual.

- Objetivos: mostrar el procedimiento de administración de anestesia realizado por una enfermera.

- Conceptos: se observa el rol asistencial que ejercían las enfermeras anestesistas.

- Comentario: se destaca la dedicación y entrega hacia el paciente, evidenciada en la administración de agentes anestésicos y el control del paciente durante la intervención quirúrgica de forma completamente manual. 


\section{DISCUSIÓN}

Las enfermeras anestesistas dedicadas únicamente a administrar anestesia fueron impulsadas por los cirujanos obteniendo mejores resultados que con personal variable (auxiliares de enfermería). Evolucionando a la creación de una subespecialidad de enfermería con capacidad de ejercicio autónomo, existiendo países donde trabajan dependientes de un anestesiólogo, y otros donde es suficiente la supervisión única del cirujano. (10)

Por otra parte, el estudio "Survey of nurse anesthesia practice, education, and regulation in 96 countries”, de M. McAuliffe y B Henry, refiere que las enfermeras anestesistas en Estados Unidos existen desde aproximadamente el año 1870, debido a, que habían muy pocos médicos por lo que las incorporaron en las prácticas quirúrgicas para que administraran anestesia. Posteriormente en 1998, bajo la supervisión de un anestesiólogo, administran alrededor del 65\% de las anestesias, y sin supervisión médica administran aproximadamente $75 \%$ de las anestesias en hospitales rurales. La administración de anestesia por enfermeras ocurre alrededor de 107 países, en algunos de los cuales ellas administran casi el $90 \%$ de estas. A pesar de esto, de ejercer el rol de anestesistas y de su contribución a la asistencia sanitaria, no han sido reconocidas por sus gobiernos, no existe documentación escrita que demuestre el rol ejercido. (11)

Es así como, se destaca la importancia que tuvieron a nivel de salud en Chile, las enfermeras anestesistas, demostrando entrega y compromiso en su labor, siendo capaces de formar otras profesionales en su área para ampliar éste círculo y garantizar la seguridad de los pacientes que tuvieron a cargo, y sobre todo de permitir que se desarrollara la cirugía en el país. Estas profesionales a pesar de haber sido subvaloradas y poco reconocidas, se desenvolvieron en un área médica con alto grado de profesionalismo, demostrando entereza, vocación y compromiso con el paciente, su profesión y la institución.

\section{CONCLUSIONES}

Las enfermeras anestesistas han sido poco reconocidas, a pesar de la importancia en el desarrollo de la cirugía en el transcurso de la historia, (11) gracias a ellas fue posible la realización de una diversidad de intervenciones quirúrgicas.

Por otra parte, el rol asumido demuestra el grado de responsabilidad y compromiso en su trabajo, capacitándose en un área que no era la suya, con el objetivo de desarrollar la cirugía en hospitales de nivel secundario y terciario donde no habían profesionales médicos especializados en dicha materia.

Por otro lado, una limitación en la realización de ésta investigación fue no contar con suficientes testigos vivenciales (fallecidas en su mayoría) y escaso material bibliográfico relacionado con el tema. 
Solo fue posible contactarse con dos enfermeras de la época, quienes colaboraron con las entrevistas, y el material fotográfico. 


\section{BIBLIOGRAFÍA}

Rodríguez Yunta E. Comités de evaluación ética y científica para la investigación en seres humanos y las pautas cioms 2002. Acta bioeth [Internet]. 2004 [consultado el 04 de diciembre de 2017]; 10(1) 37 48. Disponible en: http://www.scielo.cl/scielo.php?script=sci_arttext\&pid=S1726$569 \times 2004000100005$

Sociedad de Anestesiología de Chile SAC [Internet]. Santiago: Sociedad de Anestesiología de Chile; [consulta 29 de Abril de 2017]. Disponible en: https://www.sachile.cl/index/\#sobre-anestesia.

Chacón R. Formación de anestesista en Chile. Cómo ha evolucionado la formación en nuestra especialidad en los últimos 40 años. Rev chil anest [Internet]. 2014 [consultado 27 de agosto de 2017]; 43(3). Disponible en: http://revistachilenadeanestesia.cl/formacion-de-anestesistas-en-chile-como-haevolucionado-la-formacion-en-nuestra-especialidad-en-los-ultimos-40-anos/

Delgaso García G. Conceptos y metodologías de la investigación histórica. Revista Cubana de Salud Pública [Internet]. 2010 [consulta el 07 de julio de 2017]; 36(1) 9-18. Disponible en: http://www.redalyc.org/articulo.oa?id=21416134003

Ray W, Desai S. The history of the nurse anesthesia profession. J Clin Anesth [Internet]. 2008 [consulta el 30 de Abril de 2017]; 30 (1) 51-8. Disponible en: http://ezproxy.ufro.cl:2165/ehost/detail/detail?vid=3\&sid=88c541c5-49d3-4053-becc3b55469ed05f\%40sessionmgr104\&hid=130\&bdata=Jmxhbmc9ZXMmc210ZT1laG9zdC1saXZl\#db= mdc \&AN=27041264

Congreso Historia Enfermería 2015 [Internet]. Congreso Historia Enfermería 2015; 2016 [consulta el 29 de Abril de 2017]. Disponible en: http://www.congresohistoriaenfermeria2015.com/define.html

Vélez G. Desarrollo de la Enfermería en Anestesia en el Continente Americano. Enferm anest-reanim ter dolor [Internet]. 1998 [consulta el 26 de julio de 2017]; 6(4). Disponible en: http://www.aseedartd.org/sites/default/files/REVISTA-6-1998.pdf

ResearchGate [Internet]. Berlin: ResearchGate; 2008 [consulta el 01 de Mayo de 2017]. Disponible en: https://www.researchgate.net/profile/Jaume_Canet/publication/237217414_Encuesta_sobre_las_funcio nes_de_enfermeria_en_Anestesiologia_Reanimacion_y_Tratamiento_del_Dolor_en_Cataluna_Analisi s_de_la_situacion_actual/links/00b7d532fd63c7629b000000.pdf

Rodríguez G, Gil J, García E. Metodología de la investigación cualitativa. 1ªed. Málaga: Aljibe; 1996. American Association of Nurse Anesthetists AANA [Internet]. Illinois: AANA; 2017 [consulta el 01 de Mayo de 2017]. Disponible en: https://www.aana.com/newsandjournal/Documents/countries_1096_p469.pdf

McAuliffe M, Henry B. Survey of nurse anesthesia practice, education, and regulation in 96 countries. AANA J [Internet]. 1998 [consulta el 03 de Mayo de 2017]; 66 (3) 273-86. Disponible en: http://ezproxy.ufro.cl:2165/ehost/pdfviewer/pdfviewer?vid=4\&sid=4c4b3a1c-010c-4633-baa18c76448a08a8\%40sessionmgr103\&hid=130 